\title{
Adaptive Clipping for PAPR Reduction in OFDM
}

\author{
Vinaykumar S.Nagalikar ${ }^{1}$, Uday Pandit Khot $^{2}$ \\ ${ }^{1}$ Electronics and telecommunication, St.Xavier's Technical Institute, Mumbai, India \\ ${ }^{2}$ Uday Pandit Khot, Member IEEE, Professor, Electronics and Telecommunication Engineering Department, \\ Borivali, Mumbai, India
}

\begin{abstract}
A new Technique "Adaptive Clipping" is introduced to reduce the Peak to Average Power Ratio (PAPR) of OFDM system efficiently for wireless applications. In this technique, the threshold for clipping the signal is decided according to the peak size and number of peaks in the OFDM burst signal. A comparison is done between selective clipping and adaptive clipping and the results are shown in terms of Bit error rate (BER) and PAPR. Adaptive clipping offers nearly half a $\mathrm{dB}$ reduction in PAPR compared to selective clipping. With proposed adaptive clipping, PAPR of $3 \mathrm{~dB}$ is obtained with BER of $10^{-4}$. For a particular set of signals where there are large number of peak variations, adaptive clipping is more efficient in terms of BER compared to selective clipping. The simulation of adaptive clipping is done with the MATLAB software and the BER is plotted. An IEEE 806.11 standard is used for OFDM signal Transmission.
\end{abstract}

Keywords: BER ,OFDM, PAPR.

\section{INTRODUCTION}

THE OFDM transmission Technique is a successful technique for high speed Data communication in wireless environment. Due to its orthogonal property the interference between the subcarriers is reduced. Frequency division multiplexing allows a single high rate data to be broken in to various low speed data rates which mitigate the fading of the signal [1]. OFDM is extensively used in the data communication in Wireless applications such as wireless LAN, 4G, WIMAX, Digital (DVB) etc. The main drawback of the OFDM system is the High Peak to Average power ratio (PAPR). High PAPR results due to high change of magnitude and phase of the adjacent carriers and inability of the High power amplifier (HPA) to cope up with these along maintaining high efficiency. The non-linearity of the HPA would distort the OFDM signals; hence on the expense of efficiency non linearity of the amplifier is avoided.

The OFDM transmission is a multi-carrier transmission system and has a several advantages compared to single carrier. In OFDM several carrier signals of lower data rates run parallel. To avoid inter carrier interference the carrier are orthogonally transmitted. Each sub-carrier is modulated with digital modulation techniques such as (BPSK, QAM, and PSK ETC). The main advantages of OFDM signals are that they overcome channel conditions like interference in the signal. Frequency selective fading is overcome because the OFDM signal consists of several sun-carriers of different frequencies. Multipath fading is also overcome. The guard interval also helps the signal to provide spacing between the subcarriers. The OFDM signal is robust against narrow band co channel interference. Thus the OFDM signals are sensitive to the Frequency synchronization problems. The signals are also sensitive to Doppler shift. The major disadvantage is high Peak to average Power ratio (PAPR). High PAPR causes the HPA (High power amplifier) to go in to non-linear region of HPA which causes distortions in the signals [2]. Hence methods have been developed to overcome the high PAPR problem.

Several techniques are available for reducing PAPR among them which are commonly used for the practical is given in [3]-[6]. Inter carrier interference (ICI) is overcome by orthogonal signals, placing the modulated carriers closely increases the spectral efficiency of the OFDM system [6].

The clipping method is the simplest of all and is used extensively. In this method the signal above the fixed threshold is clipped so as to reduce PAPR. Clipping process generates in band and out of band distortion. To mitigate the distortion caused by clipping filtering technique is used after clipping. In filtering technique, convolution of the signal is performed. Clipping and filtering is widely used methods for reducing PAPR. To achieve low PAPR further iterative clipping and filtering has been proposed in [7].

This paper gives adaptive clipping where the threshold value of clipping is adapted according to the magnitude of the signals and is not always fixed. The time-domain representation of an OFDM symbol with $N$ subcarriers can be represented as follows: 


$$
s(t)=\frac{1}{\sqrt{K}} \sum_{k=0}^{K-1} X_{k} e^{j 2 \pi k t / K}, \quad 0 \leq t<T
$$

where $T_{s}$ is the duration of an OFDM signal, and $f_{k}=$ $k$

Here, all carriers are modulated with a single modulation technique. The $N$ is the number of sub carriers.

\section{PEAK AVERAGE POWER RATIO}

PAPR is the most significant problem in multi-carrier system. Several methods have been developed to overcome high PAPR. The PAPR reduction methods like clipping and filtering, selective mapping, coding, companding, probabilistic methods have been introduced The PAPR reductions techniques have a trade-off between reducing PAPR and reducing BER (Bit error rate)

The PAPR of the OFDM signal is given by

$$
\mathrm{PAPR}=\max 0 \leq \mathrm{t}<\mathrm{NT} \frac{|\mathrm{x}(\mathrm{t})|^{2}}{\mathrm{E}\left[|\mathrm{x}(\mathrm{t})|^{\mathbb{2}}\right]}
$$

\section{SYSTEM MODEL}

A block representation of an OFDM system [3] is shown in Fig 1. An OFDM system starts with the input of data stream from the communication device. The input data stream is in binary format ' 0 ' and ' 1 '. The data is modulated using any modulation sachem such as BPSK, QPSK, and QAM etc...

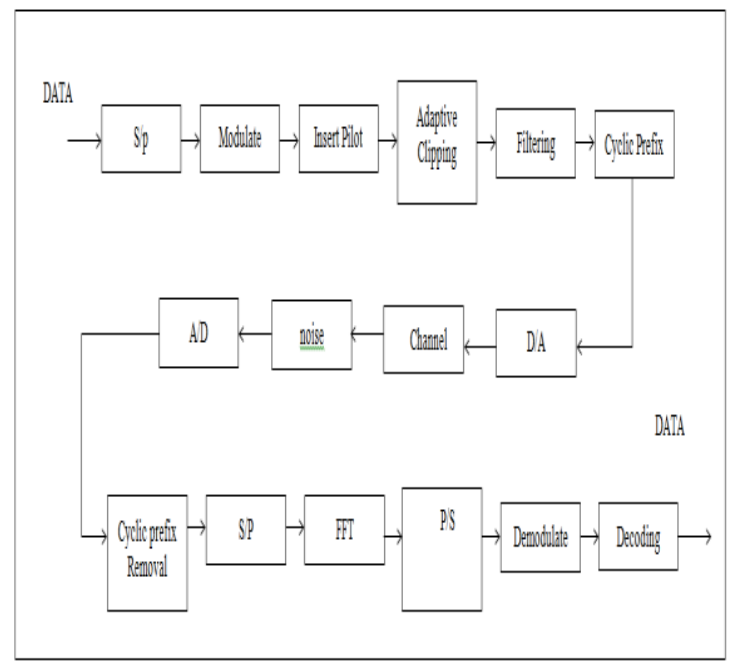

Fig 1. OFDM Block diagram representation

In our simulation QAM modulation is used. Accordingly the scatter plot is drawn. Then the data is transformed in to time domain using IFFT operation. The IFFT values are complex numbers indicating the phase and amplitude of the signal. The pilot signals are inserted according. Zero padding is used as a guard interval between the signals. The adaptive of the clipping is determined by the number of peaks exceeding the predetermined number, in which case if the peaks exceed then they are clipped at $10 \%$ of its value. The flow chart of the adaptive algorithm is shown below The OFDM communication is shown in the block diagram below. The data to be transmitted is in serial stream. The data is first broken down to $\mathrm{N}$ parallel stream as to match with the number of symbols. Again the symbols are broken down in to corresponding alphabet size for the modulation of the data. The modulation scheme such as (BPSK, QPSK, QAM, and PSK) is performed on the bits and constellation chart is prepared and are matched with this chart. The carriers can be modulated with multiple modulation schemes, so the alphabet size of the subcarrier varies according with the modulation scheme used [7].

The Inverse Fast Fourier Transform (IFFT) is performed on each set of symbols to get a time domain signal. In IFFT operation frequency domain signals are converted in to time domain signal by Discrete Fourier Transform (DFT). The IFFT signal is composed of Real and Imaginary parts. The Guard signals are inserted between the carriers to avoid interference between the sub carriers. The pilot signals are placed strategically in between the sub carrier to read the channel conditions and estimate the channel conditions.

The clipping of the Signal takes place to reduce PAPR. The Adaptive clipping technique is explained in detail in the next part. The Filtering of the signal is performed to reduce in band and out of band distortion during the clipping process. This is done by simply taking the Convolution of the signal. Then cyclic prefix is performed on the data stream for error checking. The OFDM is passed through a wireless channel and various types of noises get added with the signal [8].

OFDM Transmission is accepted world wide for high speed dta transmission and applications, Due to its increased use fine tunning of the ofdm signals are required to overcome its some of its drawbacks.the important of all is Peak to average power ratio 


\section{PROPOSED ADAPTIVE CLIPPING TECHNIQUE}

The flow chart shown in Fig. 2 explains proposed adaptive method for PAPR reduction.

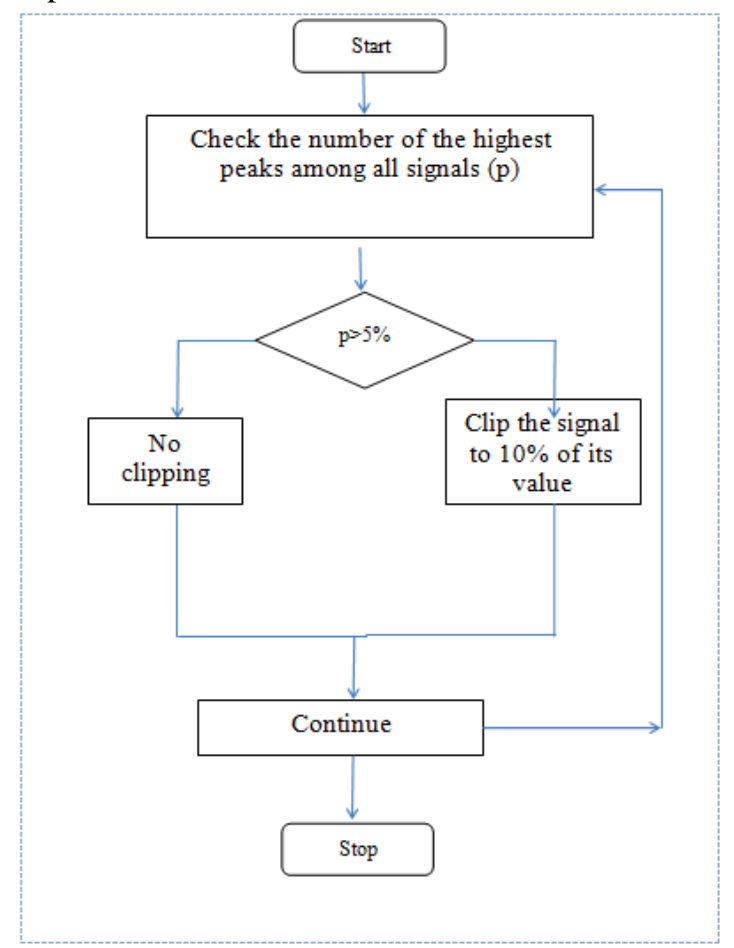

Fig. 2 The Flow Chart of Adaptive Clipping

In adaptive clipping technique, the number of the highest peaks among the signal is calculated then decision is taken to whether clip the signal or not. In contrast to clipping technique which clips the signal at $70 \%$ of the maximum value which results in high BER. As shown in the algorithm, first the signals are checked for the highest amplitude, then the signals around Adaptive clipping is very much helpful when the peaks in the signals are too many. Clipping the signals would result to considerably loss of signal and high error rate. Adaptive clipping in the other hand first checks for the number of peaks and then decision is taken to whether clip or not to clip.

If clipping is to be performed then at what level the signal is to be clipped so that there is no considerably loss of signal is calculated. Then the signal is clipped in such a way so as to maintain low PAPR in the signal. The clipping of the signal is done by the following method carefully. Firstly the highest peak in the ofdm signal is calculated, then

$10 \%$ of that value is searched. If the number of the signals under the $10 \%$ of the max value is under $5 \%$ in terms of $\mathrm{N}$ then the signal is clipped to $10 \%$ of the highest amplitude. Here some information is lost due to clipping but the PAPR is maintained as a good level. In other case if the signals are more than $5 \%$ then the signals are not clipped so as to retain the information in the signal. Here PAPR in this case may be high.

At the receiver side exact reverse operation takes place and the data is extracted back.For a particular set of signals the adaptive clipping have a less BER compared to Clipping. If the number of peaks are high then the adaptive clipping offers less BER. This happens because the clipping threshold has been kept only at $10 \%$ of the signals. If the signals are above the $10 \%$ value then the signals are not clipped to avoid significant loss of the signal.

Adaptive clipping further helps in the reduction of PAPR and improves BER. The comparison between the Clipping and adaptive clipping is done and the results are simulated in the MATLAB software.

\section{v. CONCLUSIONS}

The proposed Adaptive Clipping is more efficient than selective clipping method. The adaptive technique first checks whether the signal has to be clipped or not on the basis of the information loss in the signal. This results in improved BER. The adaptive nature of clipping is thus proved beneficial and can be used for OFDM transmission system. Adaptive clipping offers nearly half a $\mathrm{dB}$ reduction compared to selective clipping. With proposed technique, PAPR of $3 \mathrm{~dB}$ is obtained with BER of $10^{-4}$. The reductions in PAPR with improved BER have been verified for number of runs of Adaptive and selective clipping.

\section{REFERENCES}

[1] J. Bingham, "Multicarrier modulation for data transmission: an idea whose time has come," IEEE Commun. Mag., vol. 2 no. 5, pp. 5-14, May 1990.

[2] D. Dardari, V. Tralli, and A. Vaccari, "A theoretical characterization of nonlinear distortion effects in OFDM systems," IEEE Trans. Commun.,vol. 48, no. 10, pp. 17551764, Oct. 2000.

[3] S. Y. L. Goff, B. K. Khoo, C. C. Tsimenidis, and B. S. Sharif, "A novel selected mapping technique for PAPR reduction in OFDM systems,"

[4] X. Huang, J. Lu, K. B. Letaief, and J. Gu, "Companding transform for reduction in peak-to-average power ratio of OFDM signals," IEEE Trans. Wireless Commun., vol. 3, no. 6, pp. 2030-2039, Nov. 2004.

[5] X. Zhu, T. Jiang, and G. Zhu, "Novel schemes based on greedy algorithm for PAPR reduction in OFDM systems," IEEE Trans. Consum. Electron., vol. 54, no. 3, pp. 1048-1052, Aug. 2008. 
[6] J. Tong, P. Li, Z. Zhang, and V. K. Bhargava, "Iterative soft compensation for OFDM systems with clipping and superposition coded modulation," IEEE Trans. Commun., vol. 58, no. 10, pp. 28612870. Feb. 2002.

[7] J. Armstrong, "Peak-to-average power ratio reduction for OFDM by repeated clipping and frequency domain filtering," Electron. Lett., vol. 38, pp. 246-247, Oct. 2010.

[8] Malhar Chauhan, Saurabh patel, Hardik patel " Different Techniques to Reduce the PAPR in OFDM System”, International Journal of Engineering Research and Applications (IJERA) ,Vol. 2, Issue 3, pp.1292-1294, MayJun 2013 